\title{
¿Cuándo es demasiado pronto y cuándo demasiado tarde para la cirugía en la enfermedad de Crohn?
}

\author{
J. I. Fernández-Blanco Hernáiz y J. M. Monturiol Jalón ${ }^{1}$ \\ Servicio de Cirugía General y Aparato Digestivo. Hospital Universitario La Paz. Unidad de Enfermedad Inflamatoria \\ Intestinal. Clínica Moncloa. ${ }^{1}$ Hospital General Universitario Gregorio Marañón. Madrid
}

\section{RESUMEN}

El abordaje quirúrgico de la enfermedad de Crohn (EC), entendido como último esfuerzo de tratamiento frente a modalidades en las que la terapia médica ha fracasado, supone una pérdida de perspectiva, que puede posponer el retraso en la recuperación del paciente y le retrae de una mejor calidad de su vida, cuando se considera que un $50 \%$ de los pacientes mantienen inactiva su enfermedad durante años tras procedimientos quirúrgicos seleccionados; unas tasas no alcanzadas por los tratamientos más eficaces.

El riesgo de precisar algún procedimiento quirúrgico en el curso de la EC alcanza al $75 \%$ de sus portadores, más del $50 \%$ en el primer año desde el diagnóstico, y prácticamente el 100\% de los pacientes en la evolución de su proceso cuando se contemplan lesiones de localización perianal. Es por eso que el gastroenterólogo debe estar entrenado en la selección de quién, cuándo y por qué debe ser operado.

Retrasar la cirugía a fases de enfermedad avanzada incrementa la morbilidad y, si es cierto que las actuales terapias médicas permiten una mayor tasa de remisiones, también lo es que incrementar la duración del proceso y la edad de los pacientes contribuye a mayor riesgo quirúrgico y peor perspectiva en el tratamiento de complicaciones agudas y de manifestaciones crónicas de la EC, a menudo clínicamente desconsideradas, como la mesenteritis retráctil, los estados de hipercoagulabilidad y la aparición de fenómenos de malignización.

Salvando las indicaciones absolutas de actuación quirúrgica inicial en el manejo de pacientes con EC, como sangrado intestinal masivo, desarrollo de megacolon tóxico o perforación libre; otros condicionantes para el tratamiento quirúrgico deben ser reevaluados a la luz de nuestros conocimientos actuales. La genotipación del paciente constituye un elemento clínico que contribuye a identificar riesgos específicos y facilita la selección terapéutica. Desgraciadamente, hasta que estos análisis puedan ser utilizados de manera rutinaria, el empleo precoz de la cirugía en EC deberá basarse en la consideración de datos clínicos.

Diferenciar entre estenosis inflamatoria o fibrosa resulta crucial para la elección de su tratamiento, pues la cirugía supone aceptar su irreversibilidad y obliga a conjugar las manifestaciones clínicas de obstrucción crónica: dolor, distensión sobrecrecimiento bacte-

\begin{abstract}
The surgical boarding of Crohn's disease (CD) admitted as a last effort of treatment against behavior in those the therapy prescribes it has failed, it supposes a loss on perspective that can postpone the delay in the recovery of patients and it retracts them of a better quality of life when it is considered that $50 \%$ of patients maintain inactive illness during years after selected surgical procedures; some rate no reached by the most effective treatments.

The risk to specify surgical procedure in the course of $\mathrm{CD}$ rises to $75 \%$ of payees, more than $50 \%$ in the first year from the diagnosis, and practically $100 \%$ patients in the evolution when it is contemplated to attend perianal lesions. Therefore gastroenterologist should be trained in the selection who, when and why these patients should be operated. To retard the surgery to advanced illness phases increases morbidity, and if it is certain that the new biological therapy allow induction of remissions it is also it that to increase the duration of the process and the patient's age and contributes to face bigger surgical risk and worse perspectives in the treatment of their acute complications and also chronic manifestations often clinically inconsiderate as: Retractile mesenteritis, the states of hipercoagulability and the appearance of malignizations phenomena.

Saving absolute indications for initial selective surgery in management of $\mathrm{CD}$ patient like: Massive intestinal bleeding, toxic megacolon or free perforation, other surgical conditions they should be reevaluated on light of our current knowledge. Patient's genotyping constitutes a clinical element that contributes to the identification of its specific risks and it facilitates the therapeutic selection. Unfortunately until these analyses can be routinely used the precocious employment of $\mathrm{CD}$ surgery it will be based on the consideration clinical data: The patient age, its nutritional state, smoking, and the necessities of steroids.

To differ among inflammatory or fibrous stenosis is crucial for the election of their treatment, because the therapeutic employment of surgery it supposes to accept its irreversibility and makes indispensable to conjugate clinical manifestations of chronic obstruction: Pain, distension bacterial overgrowth and hipoalbuminemia with demonstration of their aperistaltic character, local hipovascularity or the submucosae muscularization submucosa in the stenosis.
\end{abstract}

\footnotetext{
Recibido: 30-07-07.
}

Aceptado: 01-09-07.

Correspondencia: J. I. Fernández-Blanco Hernáiz. Servicio de Aparato Digestivo. Hospital Universitario La Paz. Pº de la Castellana, 261. 28046 Madrid. email: fernandezblanco@telefonica.net 
riano e hipoalbuminemia con la demostración de su carácter aperistáltico, hipovascularización local y/o muscularización submucosa en el seno de la estenosis.

Por otra parte, el tratamiento médico de la participación fistulosa de la EC en los tramos proximales ofrece resultados no aceptables y esta condición debe ser asumida como indicación absoluta para el tratamiento quirúrgico. Además, los métodos diagnósticos de imagen no ofrecen una eficacia absoluta en la caracterización de ciertas modalidades penetrantes, en las cuales la eficacia de los nuevos biológicos resulta controvertida para recomendar su manejo terapéutico, por lo que la opción quirúrgica parece adquirir también una significación prioritaria en condiciones como fístulas entero-cólicas y vesicales.

El examen quirúrgico practicado bajo anestesia, con caracterización anatómica de las lesiones de la enfermedad perineal, demanda una asistencia quirúrgica precozmente realizada y se constituye en el patrón oro de definición de la enfermedad, que puede ser complementada con técnicas de imagen. Tras esta caracterización, las manifestaciones perineales de la EC deben ser asistidas, considerando la presencia o ausencia de proctitis y el fenotipo de la enfermedad subyacente. Se ha propuesto estratificar los pacientes como: portadores de fístulas simples sin proctitis, afectos de fístulas simples con enfermedad rectal asociada o portadores de fístulas complejas. Los del primer grupo serán candidatos a una terapia antibiótica e inmunosupresora de inicio; el segundo grupo a protocolos de terapia biológica añadida al programa anterior; $y$ el tercer grupo a exploración quirúrgica previa a cualquier actuación médica posterior.

El riesgo relativo acumulado a los diez años desde el diagnóstico de la EC es relativamente bajo (3\%) para el desarrollo de un cáncer colorrectal, pero asciende hasta el 33,2\% $(15,9-60,9)$ en el intestino delgado, con tasas no modificadas en los últimos 30 años, que acentúan la necesidad de programas de seguimiento o de indicación de cirugía ante su mera sospecha dado el pobre pronóstico posterior de estos pacientes.

Palabras clave: Enfermedad inflamatoria intestinal. Enfermedad de Crohn. Colitis ulcerosa. Cirugía.
On the other hand, the medical treatment of fistulous behaviors $\mathrm{CD}$ in proximal gastrointestinal tracts offers results that they cannot be considered valid and this condition should be assumed as absolute indication for the surgical treatment. The image methods diagnoses also they don't offer absolute effectiveness in the characterization on penetrating modalities in which the effectiveness of the new ones biological it is controversial to recommend its therapeutic handling for what the surgical option seems to acquire a high-priority significance under conditions as coloenteric and colovesical fistulae.

The under anesthesia surgical exam for anatomical characterization of lesions in perineal CD it demands a surgical attendance precociously carried out and it is constituted in the gold standard for definition that can be supplemented with image techniques. After characterization perianal CD they should be attended considering the presence or absence of proctitis and the phenotype of underlying illness. Some authors had intended to stratify the patients as: Simple fistula without proctitis; Affections with simple fistula associated to rectal illness; or associated complex fistulae. The first group they will be candidates to an antibiotic therapy and immunosupressors in the beginning, the second group to protocols for biological therapy added to the previous program, and the third group to previous surgical exploration to any later medical performance.

The cumulative relative risk to the ten years from the diagnosis gives the $\mathrm{CD}$ it is relatively low (3\%) for the development he/she gives a cancer colorrectal. But it ascends until 33.2\% (15.9-60.9) on the small intestine, with rate no modified in the last 30 years that accentuate the necessity of screening programs or indication for surgery against their mere suspicion the poor later presage he/she gives these patient.

Key words: Inflammatory bowel disease. Crohn's disease. Ulcerative colitis. Surgery.

Fernández-Blanco Hernáiz JI, Monturiol Jalón JM. ¿Cuándo es demasiado pronto y cuándo demasiado tarde para la cirugía en la enfermedad de Crohn? Rev Esp Enferm Dig 2008; 100: 35-44.

\section{INTRODUCCIÓN}

Abordar la selección del mejor momento para una actuación quirúrgica frente a pacientes con enfermedad de Crohn (EC) supone caracterizar los candidatos, valorando sus perspectivas de mejora de la calidad de vida (QoL) obtenida tras cirugía (1), y considerar que entre el 8590\% de los pacientes intervenidos se mantienen libres de síntomas en el primer año posterior y hasta un $20 \%$ mantiene el beneficio tras 15 años de cirugía realizada con procedimientos seleccionados (2). Estas tasas, no alcanzadas por los tratamientos médicos más eficaces, acentúan la necesidad de que el gastroenterólogo no considere como objetivo primario de su asistencia liberar al paciente de la cirugía, sino conseguir su mejor estado de bienes- tar y salvar su vida; lo que exige entrenamiento en la elección de quién, cuándo y por qué debe ser intervenido; toda vez que el riesgo de precisar algún procedimiento quirúrgico en el curso de la EC se eleva al $75 \%$ de sus portadores, de los que más del $50 \%$ requerirá de la misma en el primer año desde el diagnóstico, y prácticamente el $100 \%$ de los pacientes en la evolución de su proceso, cuando se contempla asistir las lesiones que acompañan a la localización perianal.

La necesidad de intervención quirúrgica en pacientes con EC señala a un $4 \%$, en los que la cirugía aparece como la primera medida de la actuación terapéutica, un $50 \%$ que la precisarán en los seis primeros meses desde el diagnóstico y un $20 \%$ adicional antes de los tres años desde la aparición de la enfermedad (3). El análisis de re- 
gresión señala que la mayor tasa de cirugía se manifiesta entre fumadores con EC de localización ileal, elevado número de células neutrofílicas, infiltrando sus lesiones, y una modalidad fibrosante, para cuyo control se establecen pautas de terapia esteroide en los seis primeros meses desde el inicio del proceso. El análisis univariable destaca, por el contrario, que las presentaciones cólicas con sangrado, que pueden ser manejadas con terapia aminosalicilílica y que exhiben un infiltrado leucocitario con predominio linfocitario, están inversamente asociadas al riesgo de cirugía precoz.

Por otra parte, Hulten ha demostrado que intervenir quirúrgicamente en fases de enfermedad avanzada incrementa la morbilidad postoperatoria, el riesgo de sepsis y el desarrollo de enfermedad fistulosa (4); y estudios recientes demuestran una mayor tasa de inducción de remisiones y menor mortalidad obtenidas con las nuevas terapias médicas, a expensas de incrementar la edad del paciente, la duración de su proceso y la elevación, estadísticamente significativa, de la incidencia de complicaciones agudas severas: peritonitis o perforación libre (5); y crónicas: mesenteritis retráctil, hipercoagulabilidad y displasia o cáncer (6).

\section{SELECCIÓN CLÍNICA DE LOS CANDIDATOS A CIRUGÍA}

La EC constituye un complejo sindrómico en el cual una respuesta inmunológica anómala secundaria al influjo de la flora bacteriana endógena actúa sobre condicionantes genéticos del huésped y aboca al desarrollo de daño tisular. Parece posible que modificaciones genéticas aparecidas en los vectores de reconocimiento antigénico, en las señales intracelulares por ellos determinadas, o en los productos de secreción celular inducidos por estas, puedan condicionar la aparición de diferentes modalidades de la enfermedad y su abordaje terapéutico.

Las variaciones genéticas más estudiadas en presencia de EC se refieren al gen reclutador de caspasa NOD2/CARD15 y han sido asignadas a formas de presentación ileal (7), condicionando su presencia una menor expresión por las células de Paneth locales de moléculas antibacterianas del tipo defensina-5 (8), que permite un incremento de la incorporación endosomal de antígenos mediada por el factor de necrosis tumoral alfa (TNF- $\alpha$ ), constituido así en el factor esencial de la disfunción de la barrera epitelial que contribuye a incrementar la ruta transcelular para la ingesta de productos proteicos con capacidad antigénica (9), de modo que la homozigosidad para alguna de las mutaciones en CARD15, como 1007fs, predicen el asentamiento temprano de una EC ileal con comportamiento estenótico que adquiere una más frecuentemente necesidad de cirugía y alto grado de riesgo de recurrencia postquirúrgica (10).
Siguiendo las vías celulares de señalización, es posible encontrar asociación de las tres variables más comunes de NOD2 con mutaciones en el vector de reconocimiento de lipo-polisacáridos (LPS) procedentes de la pared de las bacterias gram negativas, TLR4, que contribuyen a definir el fenotipo de presentación de la EC, habiendo sido descrita en pacientes heterocigóticos para los polimorfismos TLR4-Asp299Gly y Thr399Ile la más alta incidencia de modalidad estenosante, acentuada (47\%) cuando Asp299Gly se asocia a la ausencia de variaciones en NOD2/CARD15 (11); mientras que la mayor tasa de presentaciones fistulares $(71 \%)$ se hará presente en pacientes TLR4-/NOD2+. Del mismo modo, las mutaciones en el receptor 2 del gen productor de TNF- $\alpha$, como TNFR2:1466 A/G y $1493 \mathrm{C} / \mathrm{T}$, contribuyen, con una clara significación estadística, a un mayor riesgo de presentación fistular (12).

Otros factores con implicación patogénica establecen susceptibilidad individual entre los pacientes con EC que condicionan su capacidad de fagocitación bacteriana a través de ATG16L1, para la producción de metabolitos oxigenados reactivos mediante $\mathrm{NCF} 4$, o incriminan a las células neuroendocrinas en sus manifestaciones clínicas por variables de PHOX2B (13).

La necesidad de recurrir a la cirugía se asume así ante una falta de respuesta clínica favorable a la terapia médica o al desarrollo de complicaciones concretas que pudieran ser adelantadas. En el momento actual de nuestro conocimiento la farmacogenómica nos habilita para aventurar resultados en la aplicación de recursos terapéuticos, mediante el conocimiento de factores condicionantes referidos al componente celular presente en la mucosa lesionada (14), su capacidad de producir citoquinas proinflamatorias del tipo IFN- $\gamma / \mathrm{TNF}-\alpha$, y de inducir señales intracelulares mediadas por Nfk-beta que condicionan la respuesta inflamatoria local (15). Polimorfismos en los genes productores y receptores de citoquinas, que se postulan como agentes del daño tisular y expresión de auto-anticuerpos, pudieran ser otros elementos de análisis que contribuyan a la selección de candidatos a la actuación quirúrgica. Aunque sólo en estudios numéricamente restringidos parecen existir diferencias en la respuesta a la terapia biológica en pacientes que exhiben divergencias en su población celular de mononucleares productoras de interferón alfa (IFN- $\alpha$ ) en la lámina propria (14), los niveles mucosos de Nfk-beta, homozigosidad para el genotipo Arg196Arg en el exón 6 del receptor 2 de TNF- $\alpha$ (TNFR2), la presencia de anticuerpos anticitoplasma de los neutrófilos que expresan patrón perinuclear (pANCA).

Por otra parte, la respuesta a infliximab en portadores de patrón tipo moteado SANCA parece definir a pacientes con mejores expectativas que los que exhiben patrón pANCA, quienes no diferirían de los tratados con placebo (15). Además la homozigosidad para el haplotipo 1-11-1 del gen de la linfotoxina alfa (LTA) se ha insinuado como factor potencial de identificación de grupos de peor 
respuesta a la terapia biológica (16), del mismo modo, los portadores de anticuerpos anti-Saccharomyces cerevisae (ASCA) asocian mayor riesgo de sufrir cirugía precoz por modalidades ileales o ileocólicas (17).

Así pues, la genotipación de nuestros pacientes parece poder constituir un elemento diagnóstico que contribuya tanto a la identificación de riesgos específicos, como a la selección de las opciones terapéuticas, pero hasta que estos análisis no puedan ser ampliamente utilizados, la necesidad de empleo precoz de la cirugía en EC deberá basarse en la consideración de los datos clínicos del paciente.

Desde las primeras publicaciones, la indicación para una actuación quirúrgica inicial en la EC se establece para presentaciones con sangrado masivo, desarrollo de megacolon o de colitis tóxica, como entidades diferenciadas, y frente a la perforación libre; mientras que la cirugía electiva deberá indicarse para las modalidades perforantes o no perforantes que adquieren carácter de enfermedad intratable por los procedimientos médicos (18) y ante complicaciones crónicas como la mesenteritis retráctil, los estados de hipercoagulabilidad y el desarrollo de displasia o cáncer (19).

\section{ABSCESOS INTRACAVITARIOS}

La mayor capacidad de detección por métodos de imagen y el acceso a procedimientos de drenaje poco invasivos han permitido el abordaje externo de colecciones intracavitarias. El drenaje dirigido por radiología adquiere una doble finalidad: evitar la cirugía o permitir su actuación en condiciones más favorables que permitan menor agresividad $(20,21)$.

Si inicialmente se dudaba de su indicación en presencia de abscesos complicados o múltiples, esa aprehensión ha desaparecido. Los análisis actuales (22-24) demuestran que una considerable proporción de pacientes puede beneficiarse de dicho tratamiento, que resulta de elección en casos con diagnóstico reciente, fenotipo no penetrante, y que han sido previamente manejados sin concurso de esteroides, siempre que el procedimiento se desarrolle bajo las mismas condiciones estrictas de reposo enteral asistido con NTP y antibioterapia sistémica exigidas a la cirugía (25).

Fuera de esta situación, el drenaje quirúrgico será la indicación de actuación y habitualmente se desarrolla en dos tiempos: una primera fase de lavado y drenaje de la cavidad abscesificial; y una segunda intervención en la que se procede a la resección del segmento intestinal origen de la infección.

Bajo estas premisas, los resultados de una u otra modalidad no parecen diferir en su efectividad; pero la actuación quirúrgica precoz aporta beneficio en tanto que acorta el periodo de recuperación, disminuye la tasa de recurrencia $(12 v s .56 \%)$ y hace descender la necesidad de una nueva actuación quirúrgica posterior: $12 \mathrm{vs}$. 33$50 \%(23,25)$.

\section{ESTENOSIS OBLITERANTES}

La diferenciación entre una estenosis inflamatoria reversible y su presentación fibrosa no recuperable en la funcionalidad resulta crucial en la elección de su terapéutica, toda vez que la utilización de una cirugía exerativa supone la irreversibilidad del proceso y no estaría indicada en presencia de masa puramente edematosa. La actividad inflamatoria de una estenosis se infiere de la presencia o ausencia de ulceración sobre la misma que nos indique inflamación activa, pero a menudo esta no resulta detectable con los métodos de imagen. Criterios sonográficos de estrechamiento longitudinal de la luz entérica con incremento del espesor parietal, pérdida de definición de sus capas y descenso de la ecogeneidad definen los hallazgos habituales en la EC (26). Por otra parte, parece posible establecer que el paciente de EC con manifestaciones clínicas de obstrucción crónica, como dolor, distensión abdominal, sobrecrecimiento bacteriano e hipoalbuminemia, debe ser candidato a tratamiento quirúrgico ineludible cuando exhiba datos ecográficos de la presencia de estenosis aperistáltica con dilatación proximal de la luz (27) y descenso de la vascularización parietal en el seno de la estenosis, al ser explorado mediante doppler combinado con agentes que incrementan la señal, como el Levovist ${ }^{\oplus}$ (SHU 508 ${ }^{\text {a }}$; Schering AG, Berlín, Alemania) (28).

Esta situación puede relacionarse con los datos histológicos que definen la obliteración muscular de la submucosa en el espectro histológico de la EC (29), que remeda patrones neuromusculares y vasculares del hamartoma, donde la capa muscular, habitualmente ocupada por células musculares lisas, se ve sustituida por fibroblastos sin actividad contráctil y pérdida de su vascularización (30). De este modo la muscularización submucosa sienta el criterio de definición para la indicación terapéutica, tanto frente a la utilización de agentes anticitoquinas implicados en el desarrollo rápido de síntomas oclusivos por cambios en la deposición local de colágeno en la muscularis mucosa, submucosa y la muscularis propria (31), como ante las actuaciones mecánicas locales de dilatación endoscópica o para realizar una estricturoplastia no resectiva.

Aunque el mecanismo de desarrollo de fibrogénesis en la EC no ha sido plenamente aclarado, polímeros de la pared bacteriana parecen promover el desarrollo de fibrosis por estimulación directa de los miofibroblastos en un mecanismo mediado por el estímulo para la deposición de colágeno tipo I, TGF-1, IL-1-e IL-6 $(32,33)$. Recientemente se ha informado que infliximab infrarregula la producción de factor básico y vascular endotelial de crecimiento fibroblástico en pacientes con EC, sugiriendo una actuación infrarreguladora del proceso de fibrogenésis (34) que se perdería con la suspensión de la terapia, y también un efecto supresor del TNF- $\alpha$ en la expresión de factor de crecimiento conectivo tisular (CTGF), que puede participar en el desarrollo diferido de estenosis (35). 
Diferentes opciones no quirúrgicas han sido ofrecidas en el tratamiento de la patología obstructiva de la EC, pero tienen el inconveniente de poder ser practicadas únicamente en territorios accesibles a manipulación endoscópica, tras descartar la presencia histológica de elementos displásicos. La dilatación endoscópica con balón aventura un éxito inmediato razonable del $71 \%$ en condiciones óptimas, en estenosis sintomáticas menores de $5 \mathrm{~cm}$, localizadas en territorio gastrointestinal accesible proximal, ileal, en anastomosis ileocólica previa o ubicadas en el territorio cólico. Pero las tasas de complicación hemorrágica o perforativa son del $11 \%$ y la incidencia de recurrencia en los siguientes 18 meses del $33-76 \%$, lo que le otorga escaso beneficio a largo plazo por la necesidad de reiterar el procedimiento o recurrir a la cirugía (36).

En el intento de evitar la cirugía, se han habilitado procedimientos complementarios como la miotomía de cortes radiales practicada con esfinterótomo endoscópico (37), la dilatación mediante stents metálicos de las anastomosis ileocólicas (38), que mejoran las tasas de remisión cuando se combinan con inyección local de esteroides del tipo de la triamcinolona (39) o con administración posterior de budesonida en asociación con azatioprina (40).

Frente a estas opciones, para estenosis no tratables, que deberán ser sometidas a un estricto seguimiento de despistaje de cáncer (6), se contemplan procedimientos quirúrgicos no resectivos conocidos como estricturoplastias, que pueden ser seleccionadas según la longitud del segmento estenótico: anastomosis de Mickulicz o Finney (41), o ampliables a estenosis más complejas manejadas con otros procedimientos, Michelassi (42) o Taschieri (43), admitidos en el armamentario quirúrgico como procedimientos de elección frente a estenosis aparecidas tras cirugía, en las que una nueva resección imprime riesgo de síndrome de intestino corto o en presencia de afectación difusa con múltiples segmentos afectados, pero que únicamente estarán indicadas en el tratamiento de estenosis no flemonosas, quedando proscritas ante la presencia de sepsis abdominal por abscesificación cercana, cuando se acompañan de una fístula entérica que depara perforación libre o contenida, se asocian a múltiples estenosis en un corto segmento intestinal, se acompañan de hipoalbuminemia, adquieren riesgo de dehiscencia por suturas a tensión, o se manifiestan con una localización cólica (44).

Una nueva opción de terapia quirúrgica la constituyen las técnicas resectivas, la más común de las cuales es la resección ileocólica clásica, indicada fundamentalmente en presencia de enfermedad ileocólica que manifiesta síntomas oclusivos recurrentes. Si el objetivo actual de la cirugía de la EC es mejorar la QoL y reducir el trauma operatorio manteniendo regímenes de tratamiento eficaces, la cirugía asistida por laparoscopia puede proporcionar un buen resultado estético, menores necesidades transfusionales y de desarrollo de adhesión entérica diferida, junto a mejoras en la percepción de bienestar del paciente, con lo que constituye una opción favorable que deberá excluir a los portadores de enfermedad abscesificial o fistulosa (45) y obliga a considerar que los principales factores de reconversión a cirugía abierta los constituyen los individuos fumadores, portadores de fístulas internas, tratados con esteroides y en estados nutricionales deficitarios en el momento de la actuación (46).

Existe controversia en el manejo quirúrgico y la extensión de la resección cuando el intestino grueso resulta estar afectado. Mientras algunos autores proponen colectomías segmentarias (47), el riesgo de recurrencia postquirúrgica en el colon residual tras la colectomía segmentaria alcanza tasas más elevadas (38\%) que las identificadas en el intestino delgado proximal tras el desarrollo de colectomía $(9,3 \%)$ (48). A ello se añaden otras variables clínicas que deben ser consideradas, como el periodo de tiempo transcurrido hasta la reaparición de la enfermedad o la necesidad de mantener terapia médica, que alcanzan significación estadística y dan preferencia a la colectomía, especialmente en niños en los que las tasas de recurrencia parecen superiores, que alcanzan el 58\% (49). Con estos argumentos parece recomendable una actitud quirúrgica agresiva para la EC cólica en la que se promueva la resección total en casos de afectación ileocólica recurrida en presencia de colon esplénico y transverso afectos con colon derecho normal, reservando la pan-procto-colectomía para la afección cólica difusa con participación rectal. Tan sólo cuando la porción afectada está limitada a una pequeña porción del colon izquierdo o recto podría indicarse una resección segmentaria en aras a respetar porciones absortivas considerables que deparen diarrea.

\section{MODALIDADES PERFORANTES}

El manejo de las fístulas internas ha cambiado sustancialmente en las últimas décadas, no sólo por el acceso médico a nuevos agentes terapéuticos, sino porque, mientras la resección quirúrgica constituía el único tratamiento hace años, en la actualidad puede ser intentada la reparación lesional con abordajes quirúrgicos menos agresivos, que exigen una cuidadosa estratificación de los pacientes, atendiendo a la repercusión clínica de sus manifestaciones, de modo que únicamente las condiciones que deparan sintomatología crónica (anemia refractaria, infecciones del tracto urinario de carácter recurrente) con deterioro de la calidad de vida precisan de tratamiento quirúrgico.

\section{MODALIDADES PERFORANTES}

El manejo de las fístulas internas ha cambiado sustancialmente en las últimas décadas, no sólo por el acceso médico a nuevos agentes terapéuticos, sino porque, mien- 
tras la resección quirúrgica constituía el único tratamiento hace años, en la actualidad puede ser intentada la reparación lesional con abordajes quirúrgicos menos agresivos, que exigen una cuidadosa estratificación de los pacientes, atendiendo a la repercusión clínica de sus manifestaciones, de modo que únicamente las condiciones que deparan sintomatología crónica (anemia refractaria, infecciones del tracto urinario de carácter recurrente) con deterioro de la calidad de vida precisan de tratamiento quirúrgico.

Dos hechos fundamentales asientan la indicación de una exploración quirúrgica en presencia de EC perforativa: en primer lugar, la dificultad diagnóstica basada datos de anamnesis, el examen físico y las técnicas habituales de diagnóstico por la imagen $(50,51)$, que contribuye a que la mayor parte de las fístulas sean diagnosticadas en el momento de la cirugía; en segundo término, el diferente comportamiento de las distintas modalidades fistulares frente al tratamiento médico, cuyas tasas de respuesta global son del $69 \%$ para las fístulas externas, distribuido en un $38 \%$ para las parietales y el $78 \%$ en las perianales, pero apenas un $13 \%$ en modalidades internas y un $11 \%$ en las mixtas (52). Únicamente en estudios no controlados son accesibles datos de terapias inmunosupresoras: azatioprina (53) o los anticalcineurínicos $(54,55)$ en presencia de fístulas internas, con peores resultados que los agentes biológicos.

Las diferentes modalidades agrupadas como fístulas aisladas $(33 \%)$, fistulización en el seno de un absceso (14\%), pseudotumor inflamatorio (14\%) y adhesión fistular interasas $(2 \%)$ conllevan la necesidad de cirugía en un $93 \%$ de los casos, bien sea como cirugía electiva (44\%) o necesaria (54\%) (56). Las manifestaciones clínicas dependerán de la localización, y la extensión del intestino interpuesto será determinante en la aparición de la sintomatología, pero en general las fístulas entero-entéricas y las ileocólicas establecidas entre el íleon distal y el ciego o el colon ascendente (que crean un corto segmento excluido) a menudo resultan asintomáticas y no precisan tratamiento quirúrgico $(57,58)$, aunque a menudo se asocian a componente inflamatorio activo que exige tratamiento médico. Por el contrario, la fístula ileosigmoidea es causa de diarrea no controlable y determina sobrecrecimiento bacteriano con déficit absortivo asociado, debido a la longitud de intestino excluido. En estos casos, la opción quirúrgica resectiva constituye el patrón oro del tratamiento y se propone la resección del intestino delgado afectado con cierre del defecto cólico asociado, mediante sutura simple o fistulectomía mecánica $(59,60)$. Dado que estas resecciones no minimizan el riesgo de recurrencia y de desarrollo diferido de intestino corto, se han diseñado modelos de reparación, establecidos sobre los patrones de las estricturoplastias, como variantes menos agresivas de actuación en la enfermedad fistular, que tendrían su indicación en el intento de conservar mayor superficie intestinal $(61,62)$.

\section{FÍSTULAS GASTRO- Y DUODENO-CÓLICAS}

La participación fistulosa en tramos proximales gastroentéricos habitualmente tiene su origen en una EC localizada en colon transverso o en las anastomosis previas ileo-cólicas, por lo que se recomienda que estas se ubiquen tan lejanas al duodeno como sea posible y protegidas por epiplón.

El tratamiento médico ofrece a menudo resultados no válidos (63), por lo que adquieren una condición de indicación absoluta de tratamiento quirúrgico. El tipo de reparación más frecuentemente propuesto es la resección cólica asociada a resección limitada a los márgenes de la lesión gástrica adyacente, pero si el defecto duodenal es largo, la mayor parte de los autores promueven la realización de una gastro-yeyunostomía de asa que circunvale el duodeno afectado. Por el alto grado de probabilidad de desarrollo de ulceración secundaria en el asa (64), requiere de una vagotomía asociada que, considerando el riesgo de inducir diarrea, se propone realizar mediante técnica de vagotomía supraselectiva para conservar la inervación autonómica del delgado (65) y añadir protección del segmento duodenal mediante omentoplastia.

\section{FÍSTULAS COLOYEYUNALES}

Las fístulas ileosigmoides constituyen el tipo más común de fístulas de la EC y aparecen en el 10\% de los casos. Se ha establecido controversia en el método quirúrgico de su tratamiento, de modo que existe debate acerca de si debe realizarse resección ileocólica asociada a colectomía segmentaria (66) o una resección ileal con cierre simple del colon sigmoide (67). La exploración mediante colonoscopia realizada con anterioridad a la cirugía es obligatoria para definir la presencia de enfermedad activa local. En presencia de una afectación sigmoidea mínima, el cierre simple parece suficiente; por el contrario, si se detecta enfermedad cólica segmentaria con estenosis o fistulación múltiple, deberá realizarse resección ileo-cólica y, ante una afectación cólica difusa o universal, desarrollar una proctocolectomía (68).

\section{FÍSTULAS ENTERO-URINARIAS}

La comunicación del territorio intestinal con el aparato urinario establece unas manifestaciones clínicas patognomónicas de fecaluria, neumaturia, disuria y rasgos febriles, pero a menudo los afectados no presentan un cuadro tan florido y las exploraciones complementarias pueden resultar escasamente efectivas en identificar la lesión originaria que puede asentar en cualquier territorio intestinal: el íleon $(64 \%)$, colon $(21 \%)$ o recto $(8 \%)(69)$, que hasta en un $60 \%$ de casos se acompañan de una fístula ileosigmoide concomitante (70) y precisan estudios complementarios de confirmación diagnóstica ante la sospe- 
cha clínica de la misma y para la caracterización del territorio anatómico afectado. Hasta el acceso sistematizado a las técnicas de tomografía computerizada y de resonancia magnética la sensibilidad diagnóstica resultaba insatisfactoria, no alcanzando a identificar el proceso en la mayor parte de los pacientes mediante el enema de bario (20-50\%), la cistografía (11-30\%), la cistoscopia (44$46 \%)$ o la colonoscopia $(0-16 \%)(71)$. Este hecho ha conducido a la utilización de verde de indocianina, cuantificación de ácido acético en orina centrifugada mediante test de Bourne (72) o la determinación de componentes fecales nanomolares mediante poppy test, que alcanzan una alta sensibilidad (90-100\%) en la identificación de los pacientes afectos (65) y acentúan la necesidad de realizar estudio colonoscópico para el despistaje de enfermedad tumoral asociada a las estenosis cólica (73).

La elección del momento idóneo para la cirugía puede ser problemática cuando se asocian fístula vesical y absceso pélvico y, si la terapia antibiótica puede reducir la sintomatología, el retraso en la corrección quirúrgica puede comprometer la salud posterior del paciente, haciéndose necesaria la utilización de análisis de decisión mediante modelos compartimentales tiempo-dependientes (74).

El modo de actuación quirúrgica ha evolucionado desde la resección del intestino afectado y la reparación del defecto vesical propuesto inicialmente (75), hasta la práctica actual de resecar el intestino afecto e instaurar un catéter de Fooley de descompresión vesical hasta obtener la curación de la lesión vesical.

\section{EC PERIANAL}

El diagnóstico de la participación perianal de la EC (ECPA) resulta crucial para seleccionar la terapia médica más adecuada y requiere del clínico especial atención en el examen anamnésico en busca de síntomas y en el físico para descartar áreas de edema, discoloración eritema, skin-tags, fisuras excéntricas y orificios fistulosos en la región perianal. La exploración digital anal es obligatoria, y permite identificar estenosis y áreas de fluctuación que señalan complicación séptica, que exigen su caracterización anatómica mediante exploración bajo anestesia complementada con métodos de imagen. Nace así la necesidad de una colaboración cerrada con cirujanos colorrectales experimentados en el manejo de la ECPA que aseguren una precisa clasificación de las fístulas y sus abscesos asociados, guía imprescindible para la toma de decisiones sobre la modalidad de la terapia a aplicar: médica, quirúrgica o combinada.

La caracterización lesional nos demanda técnicas complementarias de diagnóstico por la imagen que incluyen el examen mediante ultrasonidos (SER) (76-79) y diferentes técnicas que utilizan resonancia magnética (RMN) (80-82), aportando menor sensibilidad el examen TAC y la fistulografía, por lo que han caído en desuso. Global- mente el examen quirúrgico bajo anestesia (EUA) con cateterización mediante sondas de los tractos fistulosos constituye el patrón oro de comparación y la conjunción de EUA y SER o RMN parece aportar índices de caracterización anatómica cercanos al 100\% de sensibilidad diagnóstica.

Tras su definición anatómica, las manifestaciones perianales de la EC deben ser asistidas considerando la presencia o ausencia de proctitis y enfermedad subyacente. Se ha propuesto estratificar los pacientes en tres grupos: a) portadores de fístulas simples sin proctitis; b) fístulas simples con enfermedad rectal concomitante; y c) pacientes afectados de fístulas complejas. Los pacientes del primer grupo deberían ser sometidos a terapia antibiótica e inmunosupresora de inicio, el segundo grupo a protocolos de terapia biológica añadida al programa anterior, y el tercer grupo a exploración bajo anestesia de definición quirúrgica previa a cualquier actuación médica posterior (83).

Siguiendo la clasificación de Hughes (84), la patología local sin significación séptica, skin-tags, fisuras o hemorroides, que resultan escasamente sintomáticas, no deberían ser consideradas subsidiarias de cirugía, a menos que medien defectos de higiene local que puedan favorecer su sobreinfección. Por el contrario, las lesiones sépticas locales constituyen una indicación absoluta de exploración quirúrgica bajo anestesia, para proceder precozmente a la limpieza local y la evacuación de colecciones para evitar la fibrosis local y las pérdidas de sustancia que conducen a incontinencia. Atención especial debe dirigirse a identificar el orificio interno fistular en el territorio rectal, de modo que sea permitido evacuar su contenido mediante sedales, en el caso de orificio interno accesible, o mediante catéter tipo mushroom, en ausencia de identificación del mismo. Los ultrasonidos pueden ser usados para monitorizar el seguimiento posterior hasta la desaparición de colecciones asociadas, por su facilidad de uso y por su bajo costo frente a la RMN.

Las fístulas simples, sin proctitis acompañante, pueden ser tratadas mediante fistulotomía simple, pero las que comprometen el esfínter anal se beneficiarán del implante de sedales abandonados localmente (85). Una vez desaparecida la inflamación, este sedal puede ser ajustado para reconducir su trayecto y facilitar su migración a territorios más superficiales, impidiendo la lesión esfintérica al practicar fistulotomía diferida. En ausencia de proctitis y para fístulas altas o complejas, puede practicarse flap de avance mucoso de espesor total $(86,87)$, sellado con adhesivos de fibrina (88-90), mediante técnicas combinadas de reparación del orificio interno y relleno del tracto fistular residual con fibrina $(91,92)$, o con implante local de stem cells (93), pero las fístulas ano-recto-vaginales, que muestran peor tasa de resultados para esta técnica, deben ser reparadas.

Las estenosis anales sintomáticas deberán ser tratadas con dilatación digital, mediante bujías o con dilatación endoscópica y sometidas a vigilancia de desarrollo de 
neoplasia y, en ausencia de respuesta o sepsis perianal, asociar al tratamiento una derivación fecal o el desarrollo de protectomía (6).

\section{DISPLASIA/CÁNCER}

El riesgo de desarrollo de cáncer en portadores de enfermedad inflamatoria parece incrementado respecto a la población general, con una incidencia similar en EC y colitis ulcerosa para el desarrollo de tumores extraintestinales localizados en el sistema hepatobiliar, un mayor porcentaje de riesgo de linfoma entre las mujeres portadoras de EC y diferencias estadísticas en la incidencia de cáncer intestinal entre ambos procesos (94), que confirman que tanto a nivel colorrectal como asentado en intestino delgado las tasas de incidencia resultan incrementadas entre portadores de EC (95) y se relacionan con la duración del proceso y la severidad de la inflamación (96). Un meta-análisis cercano (97) señala niveles globales de riesgo de cáncer colorrectal de 2,5 (1,3-4,7), 4,5 (1,314,9) para los pacientes con afectación cólica y del 1,1 $(0,8-1,5)$ en presencia de enfermedad ileal. Aunque pudieran existir diferencias étnicas en la tasa de incidencia, el riesgo relativo acumulado a los diez años desde el diagnóstico de la EC es del 2,9\% (1,5-5,3\%) para el desarrollo de cáncer colorrectal y asciende hasta el 33,2\% $(15,9-60,9 \%)$ en el intestino delgado, con tasas que no se han reducido en los últimos 30 años y que acentúan la necesidad de programas de seguimiento pues, a pesar de la falta de evidencia de que los estudios endoscópicos secuenciados consigan prolongar la supervivencia de los pacientes con colitis extensa, parecen contribuir a un mejor pronóstico por su detección más precoz (98).

La mayor incidencia de adenocarcinoma asociado a EC se produce en el intestino delgado, y con preferencia en íleon final (89\%) frente al yeyuno (11\%) y en pacientes con enfermedad avanzada en el momento del diagnóstico, sin que hayan podido ser caracterizados factores de riesgo asociado (99). Se han señalado diferencias tanto por su localización entre el adenocarcinoma asentado sobre EC, que se desarrolla en áreas inflamadas ileales afectas de EC en los diez años previos, con aparición esporádica en el seno de estricturoplastias (100-102), frente al aparecido de novo sin preferencia de localización en el intestino delgado; como en su componente histológico con incremento de la presencia de células en anillo de sello (35\%) en EC y ausencia en el espontáneo (103). Las variables histológicas que establecen peor pronóstico parecen estar relacionadas con un diagnóstico tardío y se consideran homologables con las de otros carcinomas, incluyendo la presencia tumoral en los márgenes quirúrgicos, la extensión extramural o venosa de la lesión y el compromiso de los ganglios linfáticos locales, así como una pobre diferenciación y la profundidad de la lesión (104), que ensombrecen la curabilidad $(54,9,30,5$ y $27,4 \%)$ y los índices de supervivencia $(43,6,22,8$ y
$17,5 \%)$ a uno, tres y cinco años respectivamente (105).

La presencia de displasia/cáncer en el territorio cólico obliga a procedimientos quirúrgicos. En presencia de displasia aislada sobre un pólipo, este puede ser resecado mediante endoscopia y si se hace patente sobre mucosa plana practicar una resección segmentaria cólica. Las lesiones ubicadas por encima del recto pueden tratarse mediante colectomía subtotal pero las lesiones rectales aconsejan una resección abdómino-perineal.

\section{BIBLIOGRAFÍA}

1. Tillinger W, Mittermaier C, Lochs H, et al. Health-related quality of life in patients with Crohn's disease: Influence of surgical operation -a prospective trial. Dig Dis Sco 1999; 44: 932-8.

2. Peppercorn MA. UpToDate 2007.

3. Sands BE, et al. Risk of early surgery for Crohn's disease: Implications for early treatment strategies. Am J Gastroenterol 2003; 98 (12): 2712-8.

4. Nordgren SR, Fasth SB, Oresland TO, Hultén LA. Long-term follow-up in Crohn's disease. Mortality, morbidity, and functional status. Scand J Gastroenterol 1994; 29 (12): 1122-8.

5. Siassi M, Weiger A, Hohenberg W, Kessler H. Changes in surgical therapy for Crohn's disease over 55 years: A prospective longitudinal study. Int J Colorectal Dis 2007; 22 (3): 319-24.

6. Halverson A. Timing for surgery in Crohn's disease. Am J Gastroenterology 2007; 102: S79-S80.

7. Hugot JP, Chamaillard M, Zouali H, et al. Association of NOD2 leucine-rich repeat variants with susceptibility to Crohn's disease. Nature 2001; 411 (6837): 599-603.

8. Wehkamp J, Harder J, Weichenthal M, et al. NOD2 (CARD15) mutations in Crohn's disease are associated with disminushed mucosal alpha-dephensin expression. Gut 2004; 53: 1658-64.

9. Söderholm JD, Streutker C, Yamg PC, et al. Increased epithelial uptake of protein antigens in the ileum of Crohn's disease mediated by tumor necrosis factor alpha. Gut 2004; 53: 1817-24.

10. Seiderer J, Schnitzler F, Brand S, et al. Homozygosity for the CARD15 frame shift mutation $1007 \mathrm{fs}$ is predictive of early onset of Crohn's disease with ileal stenosis, entero-enteral fistulas, and frequent need for surgical intervention with high risk of re-stenosis. Scand J Gastroenterol 2006; 41 (12): 1421-32.

11. Brand S, Staudinger T, Schnitzler F, et al. The role of toll-like receptor 4 Asp299Gly an Thr399Ile polymorphisms and Card12/NOD2 mutations in susceptibility and phenotype of Crohn's disease. Inflamm Bowel Dis 2005; 11 (7): 645-52.

12. Sashio H, Tamura K, Ito R, et al. Polymorphisms of the TNF gene and the TNF receptor superfamily member $1 \mathrm{~B}$ gene are associated with susceptibility to ulcerative colitis and Crohn's disease, respectively. Immunogenetics 2002; 53: 1020-7.

13. Rioux JD, Xavier RJ, Taylor KG, et al. Genome wide association study identifies new suceptibility loci for Crohn's disease and implicates autophagy in diseases pathogenesis. Nat Genet 2007; 39 (5): 596-604.

14. Shetty A, Forbes A. Pharmacogenomics of response to anti-tumor necrosis factor therapy in patients with Crohn's disease. Am J Pharmacogenomics 2002; 2: 215-21.

15. Nikolaus S, Raedler A, Kühbacker T, Sfikas N, Fölsch UR, Schreiber S. Mechanisms in failure of infliximab for Crohn's disease. Lancet 2000; 356 (9240): 1475-9.

16. Taylor KD, Plevy SE, Yang H, et al. ANCA pattern and LTA haplotype relationship to clinical responses to anti-TNF antibody treatment in Crohn's disease. Gastroenterology 2001; 120 (6): 1347-55.

17. Forcione DG, Rosen MJ, Kisiel JB, Sands BE. Anti-Sacharomyces cerevisae antibody (ASCA) positivity is associated with increase risk for early surgery in Crohn's disease. Gut 2004; 53: 1117-22.

18. Greenstein AJ. Indications and procedures for surgery of small bowell Crohn's disease. In: Bayless TM, Hanauer SB, editors. Advanced Therapy of Inflammatory Bowel Disease. London: BC Decker Inc; 2001. 
19. Sachar DB. Indications for surgery in Crohn's disease. Am J Gastroenterol 2007; 102: S76-8.

20. Ayuk P, Williams N, Scott NA, Nicholson DA, Irvin MH. Management of intra-abdominal abcesses in Crohn's disease. Ann R Coll Surg Engl 1996; 78: 5-10.

21. Safri HD, Mauro MA, Jacques PF. Percutaenous absceee drainage in Crohn's disease. Am J Roentgenol 1987; 148: 859-62.

22. García JC, Persky SE, Bonis PA, Topazian M. Abscesses in Crohn's disease: Outcome of medical versus surgical treatment. J Clin Gastroenterol 2001; 32 (5): 409-12.

23. Gervais DA, Hahn PF, O'Neil MJ, Mueller PR. Percutaneous abscesses drainage in Crohn's disease: Technical success and shortand long-term outcomes during 14 years. Radiology 2002: 22: 64551.

24. Yamaguchi A, Matsui T, Sakurai T, et al. The clinical characteristics and outcome of intrabadomninal abscess in Crohn's disease. J Gastroenterol 2004; 39 (5): 441-8.

25. Gutiérrez A, Lee H, Sand BE. Outcome of surgical versus percutaneous drainaje of abdominal and pelvic abscesses in Crohn's disease. Am J Gastroenterol 2006; 101: 2283-9.

26. Schwerk WB, Beckh K, Raih MA. A prospective evaluation of high resolution sonography in the diagnosis of inflammatory bowel disease. Eur J Gastroenterol Hepatol 1992; 4: 173-82.

27. Parente F, Maconi G, Bollani S, et al. Bowel ultrasound in assessment of Crohn's disease and detection of related small bowel strictures: A prospective comparative study versus $\mathrm{x}$ ray and intraoperative findings. Gut 2002; 50: 490-5.

28. Kratzer W, von Tirpitz C, Mason R, et al. Contrast-enhanced power doppler sonography of the intestinal wall in the differentiation of hypervascularized and hipovascularizec intestinal obstruction with Crohn's disease. J Ultrasound Med 2002; 21: 149-57.

29. Sheperd NA, Jass JR. Neuromuscular and vascular hamartoma of the small intestine: Is it Crohn's disease ? Gut 1987; 28: 1663-8.

30. Koukoulis G, Ke Y, Henley JD, Cummings OW. Obliterative muscularization of the small bowell submucosa in Crohn's disease. Arch Pathol Lab Med 2001; 125: 1331-4.

31. Sidham VB, Komoroswki R, Asma Z, et al. Histopathologic changes in patients presenting with obstruction secondary to infliximab therapy in Crohn's disease. Mod Pathol 2001: 14: 95A (abst. 547)

32. Van Tol EAF, Holt L, Feng Ling L, et al. Bacterial cell wall polymers promote intestinal fibrosis by direct stimulation of myofibroblasts. Am J Physiol 1999; G245-5.

33. Puciloswak JB. Fibrosis in inflammatory bowel disease. Cellular mediators and animal models. Am J Physiol 2000; 279: G653-9.

34. Di Sabatino A, Ciccocciopo R, Benazzato L, et al. Infliximab downregulates basic fibroblast growth factor and vascular endotelial growth factors in Crohn's disease patients. Aliment Pharmacol Ther 2004; 19: 1019-24.

35. Beddy B, Muslow RWG, Fitzpatrick JM, O'Connell PR. Expression and regulation of connective tissue growth factor by transforming growth factor alpha and tumor necrosis factor alpha in fibroblasts isolated from strictures in patients with Crohn's disease. Br J Surg 2006; 93: 1290-6.

36. Van Asche G, Vermiere S, Rutgeerts P. Endoscopic therapy of strictures in Crohn's disease. Inflamm Bowel Dis 2007; 13 (3): 356-8.

37. Bedogni G, Ricci E, Pedrazolli C, et al. Endoscopic dilatation of anastomotic colonic stenosis by different techniques: An alternative to surgery? Gastrointest Endosc 1987: 33: 21-4

38. Matsuhashi N, Nakayima A, Suzuki A, et al. Long-term outcome of non-surgical strictureplasty using metallic stents for intestinal strictures in Crohn's disease. Gastroint Endoscopy 2000; 51: 343-5.

39. Brooker JC, Beckett CG, Saunders BP, Benson MJ. Long-acting steroid injection after endoscopic dilation of anastomotic Crohn's strictures may improve the outcome: A retrospective case series. Endoscopy 2003; 35 (4): 333-7.

40. Raedler A, Peters L, Schreiber S. Treatment with azathioprine and budesonide prevents recurrence of ileocolonic stenoses after endoscopic dilatation in Crohn's disease. Gastroenterology 1997; 112: A1067.

41. Alexander-Williams J. The technique of intestinal strictureplasty. Int J Colorectal Dis 1986; 1: 54-7.

42. Michelassi F. Side to side isoperistaltic strictureplasty for multiple Crohn's strictures. Dis Colon Rectum 1996; 39: 345-9.
43. Tascheri AM, Cristaldi M, Elli M, Danieli PG, et al. Description of new bowel-sparing technique for long strictures of Crohn's disease. Am J Surg 1997; 173: 509-12.

44. Resegotti A, Astegiano M, Sostegni R, et al. Strictureplasty in Crohn's disease. Indications and results. Minnerva Chir 2000; 55 (3): 313-7.

45. Mirow L, Schwandner O, Fischer F, Farke S, Bruch HP. Minimal invasive surgery for Crohn's disease-indication, technique and results. Eur Surg 2006; 38 (4): 302-6.

46. Schmidt CM, Talamini MA, Kaufman HS, et al. Laparoscopic surgery for Crohn's disease: Reasons for conversion. Ann Surg 2001; 233 (6): 733-9.

47. Polle SW, Slors JF, Weverling GJ, Gouma DJ, Hommes DW, Bemelman WA. Recurrence after segmental resection for colonic Crohn's disease. Br J Surg 2005; 92 (9): 1143-9.

48. Fichera A, McCormack R, Rubin MA, Hurst RD, Michelassi F. Long-term outcome of surgically treated Crohn's colitis: A prospective study. Dis Colon Rectum 2005; 48 (5): 963-9.

49. Ba'ath ME, Mahmalat MW, Kapur P, Smith NP, Dalzell AM, Casson DH, et al. Surgical management of inflammatory bowel disease. Arch Dis Child 2007; 92 (4): 312-6.

50. Broe PJ, Bayless T, Cameron JL. Crohn's disease: Are enteroenteral fistulas an indication for surgery? Surgery 1982; 91: 249-53.

51. Michelassi F, Stella M, Balestracci T, et al. Incidence diagnosis, and treatment of enteric and colorectal fistulas in patients with Crohn's disease. Ann Surg 1993; 218: 660-6.

52. Parsi MA, Lashner BA, Achkar JP, et al. Type of fistula determines response to infliximab in patients with fistulous Crohn's disease. Am J Gastroenterol 2004; 99 (3): 445-9.

53. Korelitz B, Present D. Favorable effect of 6-mercaptopurine in fistula of Crohn's disease. Dig Dis Sci 1985; 30: 58-64.

54. Present D, Lichtiger S. Efficacy of cyclosporine in treatment of fistula of Crohn's disease. Dig Dis Sci 1994; 39: 374-80.

55. Sandborn W. Preliminary report on the use of oral tacrolimus (FK 506) in the treatment of complicated proximal small bowel and fistulizing Crohn's disease. Am J Gastroenterol 1997; 92: 876-9.

56. Brihier $\mathrm{H}$, Nion-Larmurier I, Afchain $\mathrm{P}$, Tiret $\mathrm{E}$, Baeaugerie L, Gendre JP, et al. Intestinal perforation in Crohn's disease. Factors predictive of surgical resection. Gastroenterol Clin Biol 2005; 11 (29): 1105-11.

57. Liechtenstein G. Treatment of fistulizing Crohn's disease. Gastroenterology 2000; 119: 1132-47.

58. Yamamoto T, Bain IM, Connolly AB, et al. Gastroduodenal fistulas in Crohn's disease: Clinical factors and management. Dis Colon Rectum 1998; 41 (10): 1287-92.

59. Poritz LS, Gagliano GA, McLeod RS, MacRae H, Cohen Z. Surgical management of entero and colocutaneous fistulae in Crohn's disease: 17 years' experience. Int J Colorectal Dis 2004; 19 (5): 481-5.

60. Ikeuchi H, Shoji Y, Yamamura T. Management of fistulas in Crohn's disease. Dig Surg 2002; 19 (1): 36-9.

61. Poggioli G, Selleri S, Stocchi L, Laureti S, Salone M, Marra C, et al. Conservative surgical management of perforating Crohn's disease: Side-to-side enteroenteric neoileocolic anastomosis: Report of two cases. Dis Colon Rectum 1998; 41: 1577-80.

62. Poggioli G, Pierangeli F, Laureti S, Ugolini F. Review article: Indication and type of surgery in Crohn's disease. Aliment Pharmacol Ther 2002; 16 (Supl. 4): 59-64.

63. Greenstein A, Present D, Sachar D, et al. Gastric fistulas in Crohn's disease. Report of cases. Dis Colon Rectum 1989; 32: 888-92.

64. Ross TM, Fazio VW, Farmer RG. Long-term results of surgical treatment for Crohn's disease of the duodenum. Ann Surg 1983; 197: 399-406.

65. Marcello PW, Schoetz DJ Jr. Gastroduodenal Crohn's disease: Surgical management. In: Bayless TM, Hanauer SB, editors. Advanced therapy of Inflammatory Bowel Disease. Hamilton, London: BC Decker Inc.; 2001. p. 461-3.

66. Fazio V. The dilemma of Crohn's disease: Ileosigmoidal fistula complicating Crohn's disease. Dis Colon Rectum 1977; 20: 381-6.

67. Levy C, Tremaine WJ. Management of internal fistula in Crohn's disease. Inflam Bowel Dis 2002; 8 (2): 106-11.

68. Greenstein AJ. Indication and procedures for surgery in small bowel Crohn's disease. In: Bayless TM, Hanauer SB, editors. Advanced therapy of Inflammatory Bowel Disease. Hamilton, London: BC Decker Inc.; 2001. p. 443-7. 
69. Solem C. Fistulas to the urinary system in Crohn's disease: Clinical features and outcomes. Am J Gastroneterol 2000; 95: 2551A.

70. Annibali R. Fistulous complications of Crohn's disease. Int Surg 1992; 77: 119-27.

71. Najjar S, Jamal M, Savas J, Miller T. The spectrum of colovesica fistula and diagnostic paradigm. Am J Surg 2004; 188 (5): 617-21.

72. Amendola MA, Agha FP, Dent TL, et al. Detection of occult colovesical fistula by Bourne test. Am J Roentgenol 1984; 142: 7158.

73. Garcea G, Majid I, Sutton CD, Pattenden CJ, Thomas WM. Diagnosis and management of colovesical fistulae; Six-year experience of 90 consecutive cases. Colorectal Dis 2006; 8 (4): 347-52.

74. Sonnenberg A. A timing of surgery for enterovesical fistula in Crohn's disease. Inflamm Bowel Dis 2000; 6: 280-5.

75. Hyen F. Enterovesical fistula in Crohn's disease. Am R Coll Surg Engl 1989; 71: 101-4.

76. Felt-Bersma RJ. Endoanal ultrasound in perianal fistulas and $a b-$ scesses. Dig Liver Dis 2006; 38 (8): 537-43.

77. Schwartz DA, White CM, Wise PE, Herline AJ. Use of endoscopic ultrasound to guide combination medical and surgical therapy for patients with Crohn's perianal fistulas. Inflamm Bowel Dis 2005; 11 (8): 727-32

78. Caprioli F, Losco A, Viganò C, Conte D, Biondetti P, Forzenigo LV et al. Computer-assisted evaluation of perianal fistula activity by means of anal ultrasound in patients with Crohn's disease. Am J Gastroenterol 2006; 101 (7): 1551-8.

79. Orsoni P, Barthet M, Portier F, Panuel M, Desjeux A, Grimaud JC. Prospective comparison of endosonography, magnetic resonance imaging and surgical findings in anorectal fistula and abscess complicating Crohn's disease. Br J Surg 1999; 86 (3): 360-4.

80. Schäfer AO, Baumann T, Langer M. MRI for the detection of anorectal fistulas. Rofo 2006; 178 (11): 1095-149.

81. Dwarkasing S, Hussain SM, Hop WC, Krestin GP. Anovaginal fistulas: Evaluation with endoanal MR imaging. Radiology 2004; 231 (1): $123-8$

82. Schaefer O, Lohrmann C, Langer M. Assessment of anal fistulas with high-resolution subtraction MR-fistulography: Comparison with surgical findings. J Magn Reson Imaging 2004; 19 (1): 91-8.

83. Schwartz DA, Herdman CR. Review article: The medical treatment of Crohn's perianal fistulas. Aliment Pharmacol Ther 2004; 19 (9): 953-67.

84. Hughes LE. Clinical classification of perianal Crohn's disease. Dis Colon Rectum 1992; 35 (10): 928-32.

85. Beck DE. Management of anorectal Crohn's fistulas. Clinics in colon rectal surgery $2001 ; 14$ (2): 117-28

86. Ozuner G, Hull TL, Cartmill J, et al. Long-term analysis of the use of transanal rectal advanced flap for complicate anorectal/vaginal fistulas. Dis Colon Rectum 1996; 39: 10-4.

87. Sonoda T, Hull T, Piedmonte MR, Fazio VW. Outcomes of primary repair of anorectal and rectovaginal fistula using the endorectal advancement flaps. Dis Colon Rectum 2002; 45 (12): 1622-8.

88. Cintron JR, Parks JJ, Orsay CP, Pearl RK, Nelson RL, Sone JH, et al. Repair of fistulas in ano using fibrin adhesive: Long term follow up. Dis Colon Rectum 2000; 43: 944-9.
89. Chan KM, Lau KK, Auyeung MC, Ho LS, Luk HT, et al. Preliminary results of using a commercial fibrin sealant in the treatment of fistula in ano. J R Coll Surg Edinb 2002; 47: 407-10.

90. Fernández-Blanco JI, Álvarez J, Acitores I, et al. Enterocutaneous fistula in Crohn's disease. Fistulous sealing with autologous fibringlue. Gut 2002; 51 (Supl. III): A305.

91. Fernández-Blanco JI, Navajas J, Álvarez J, et al. Fistulous sealing in Crohn's disease. Gut 2004; 53 (Supl. IV): Abst. 227.

92. Vitton V, Gasmo M, Barthet M, Orsoni P, Desjeux A, Grimaud J. Is healing of perianal fistulas complicating Crohn's disease by using fibrin glue efficient and durable? Gut 2004; 53 (Supl. VI): A223.

93. García-Olmo D, García-Arranz M, García LG, Cuéllar ES, Blanco IF, et al. Autologous stem cell transplantation for treatment of rectovaginal fistula in perianal Crohn's disease: A new cell-based therapy. Int J Colorectal Dis 2003; 18 (5): 451-4

94. Bernstein CN, Blanchard JF, Kliewer E, Wajda A. Cancer risk in patients with inflammatory bowel disease. A population-based study. Cancer 2001; 91 (4): 854-62.

95. Jess T, Gamborg M, Matzen P, Munkholm P, Sørensen TI. Increased risk of intestinal cancer in Crohn's disease: A meta-analysis of population-based cohort studies. Am J Gastroenterol 2005; 100 (12): 2724-9.

96. Rutter M, Saunders B, Wilkinson K, et al. Severity of inflammation is a risk factor for colorectal neoplasia in ulcerative colitis. Gastroenterology 2004; 126: 451-9.

97. Canavan C. Meta-analysis: Colorectal and small bowel cancer risk in patients with Crohn's disease. Aliment Pharmacol Ther 2006; 23 (8): 1097-104.

98. Collins PD, Mpofu C, Watson AJ, Rhodes JM. Strategies for detecting colon cancer and/or dysplasia in patients with inflammatory bowel disease. Cochrane Database Syst Rev 2006; (2): CD000279.

99. Solem CA, Harmsen WS, Zinsmeister AR, Loftus EV. Small intestinal adenocarcinoma in Crohn's disease: A case-control study. Inflamm Bowel Dis 2004; 10 (1): 32-5

100. Marchetti F, Fazio VW, Ozuner G. Adenocarcinoma arising from a strictureplasty site in Crohn's disease. Report of a case. Dis Colon Rectum 1996; 39 (11): 1315-21.

101. Partridge SK, Hodin RA. Small bowel adenocarcinoma at a strictureplasty site in a patient with Crohn's disease: Report of a case. Dis Colon Rectum 2004; 47 (5): 778-81.

102. Menon AM, Mirza AH, Moolla S, Morton DG. Adenocarcinoma of the small bowel arising from a previous strictureplasty for Crohn's disease: Report of a case. Dis Colon Rectum 2007; 50 (2): 257-9.

103. Palascak-Juif V, Bouvier AM, Cosnes J, et al. Small bowel adenocarcinoma in patients with Crohn's disease compared with small bowel adenocarcinoma de novo. Inflamm Bowel Dis 2005; 11 (9): 828-32.

104. Abrahams NA, Halverson A, Fazio VW, Rybicki LA, Goldblum JR. Adenocarcinoma of the small bowel: A study of 37 cases with emphasis on histologic prognostic factors. Dis Colon Rectum 2002; 45 (11): 1496-502.

105. Wu TJ, Yeh CN, Chao TC, Jan YY, Chen MF. Prognostic factors of primary small bowel adenocarcinoma: Univariate and multivariate analysis. World J Surg 2006; 30 (3): 391-8. 

PARA LA CIRUGÍA EN LA ENFERMEDAD DE CROHN? 